\title{
Optimisation du procédé de pliage pour la mise en forme de pièces de sécurité automobile
}

\author{
Riadh Bahloul* — Ali Mkaddem**_ Philippe Dal Santo* \\ Alain Potiron* — Delphine Saïdane*** \\ * ERT-LPMI Ecole Nationale Supérieure d'Arts et Métiers \\ 2, bd. du Ronceray - BP 93525 \\ F-49035 Angers cedex 1 \\ ** Ecole Nationale Supérieure d'Arts et Métiers \\ rue Saint Dominique BP 508 \\ F-51006 Châlons-en-Champagne \\ *** Société DEVILLE S.A. \\ Zone industrielle Beauregard \\ F-49150 Baugé \\ alain.potiron@angers.ensam.fr
}

\begin{abstract}
RÉSUMÉ. Ce travail concerne l'optimisation d'un procédé de mise en forme de pièces de sécurité obtenues par découpage et pliage. Les études ont pour but de minimiser les contraintes et l'endommagement des matériaux ainsi que le retour élastique à la fin de la phase de pliage. Des simulations du procédé par la méthode des éléments finis, permettent de construire des tables d'expériences numériques dont l'analyse par surfaces de réponse quantifie l'influence des principaux paramètres de l'opération de pliage. Une optimisation multi-objectif permet de minimiser conjointement le retour élastique et le dommage. L'influence du choix des matériaux est analysée afin d'optimiser la conception de nouvelles pièces obtenues par pliage.

ABSTRACT. In this work, the process optimisation of security parts manufacturing is simulated by a finite element modelling including a material damage formulation. Objective functions, stress and damage values occurring in the part at the end of a bending operation, are analysed by means of numerical Design of Experiments and Response Surface Methodology. The process optimisation is carried out by global minimisation and by means of Evolution Strategies. A multi-objective optimisation is carried out in order to identify the best choice of material and process parameters giving a reliable part-life.

MOTS CLÉS: endommagement, éléments finis, optimisation, pièces de sécurité, pliage sur presse, retour élastique, stratégie d'évolution.

KEYWORDS: bending operation, damage, design of experiments, evolution strategies, F.E. modelling, optimisation, response surface, spring back.
\end{abstract}

DOI :10.3166/REMN.17.323-348 @ 2008 Lavoisier, Paris 


\section{Introduction}

La sécurité des passagers de véhicules automobiles est un enjeu essentiel de la recherche industrielle dans les industries du transport sur route sachant que les pièces de retenue des passagers telles que les ferrures d'ancrage des ceintures de sécurité, ou les boîtiers de coussins gonflables, sont fabriquées en très grandes séries (plusieurs dizaines de millions d'unités par an). La recherche d'une productivité accrue et d'une fiabilité parfaite de ces ensembles oblige les fabricants à optimiser les formes et les procédés de fabrication car la majeure partie des procédés d'obtention des pièces concerne la mise en forme par déformations plastiques sur presses à l'aide d'outils à suivre très coûteux.

Même si la forme des pièces d'ancrage des ceintures de sécurité reste inchangée depuis une vingtaine d'année, l'arrivée sur le marché d'aciers à haute limite élastique (H.L.E.) toujours plus performants incite les bureaux d'études à se pencher sur l'optimisation des formes de ces composants et sur l'optimisation des procédés.

C'est dans ce contexte que se situe l'étude présentée ici avec deux volets principaux : (i) l'optimisation du procédé de pliage sur presse avec outil à suivre, (ii) l'aide à la décision pour le choix de matériaux devant être pliés à $90^{\circ}$ sur presse.

Le découpage de tôles sur presses au moyen d'un poinçon et d'une matrice a fait l'objet de nombreuses études depuis sa mise en œuvre industrielle (Hambli et al., 2000 ; Lemiale et al., 2002; Hambli et al., 2004). Plusieurs modèles ont été proposés pour suivre les phénomènes de cisaillement conduisant à la rupture, parmi lesquels les plus populaires sont les modèles d'endommagement de Gurson et de Lemaître et Chaboche (Lemaître et al., 1988). Ce dernier modèle semble le plus approprié pour représenter l'ensemble des phases de découpage et c'est celui qui est retenu dans cette étude.

De la même manière, le pliage à $90^{\circ}$ sur presse à l'aide d'un poinçon et d'une matrice est une opération apparemment simple mais qui entraîne :

- des erreurs géométriques sur la forme des pièces, erreurs induites par un inévitable phénomène de retour élastique, sauf à utiliser des techniques appropriées comme la frappe sur l'arrondi en fin de pliage,

- une modification de la résistance de la pièce entrainée par un endommagement du matériau dans la zone pliée.

Dans les deux procédés évoqués, si on veut maîtriser les fabrications et garantir la tenue à l'usage des composants de sécurité, il est impératif de prédire l'évolution du comportement du matériau lors de sa mise en forme et ses conséquences sur les pièces formées. Un modèle analytique du pliage est donné dans (Wang et al., 1993). 


\section{Analyse et simulation des procédés}

\subsection{Description sommaire du procédé d'obtention des ferrures}

Les pièces étudiées sont essentiellement des ferrures d'ancrage de ceintures de sécurité pour véhicules automobiles. Les deux procédés décrits précédemment interviennent séquentiellement sur des bandes métalliques comme on l'a présenté sur la figure 1 pour mettre en forme ces attaches. Les bandes reçues sous forme de bobines sont déroulées, redressées puis passent dans les presses pour la mise en forme des pièces. Après formage, la ferrure d'ancrage sur laquelle est attaché le brin mort de la ceinture de sécurité, est boulonnée sur le châssis. Ce brin mort est fixé dans un trou oblong qui fragilise l'attache par des concentrations de contraintes dans son voisinage. Un trou de passage de boulon pour la fixer sur le châssis du véhicule, complète la définition géométrique de la pièce dont l'aspect général est triangulaire.
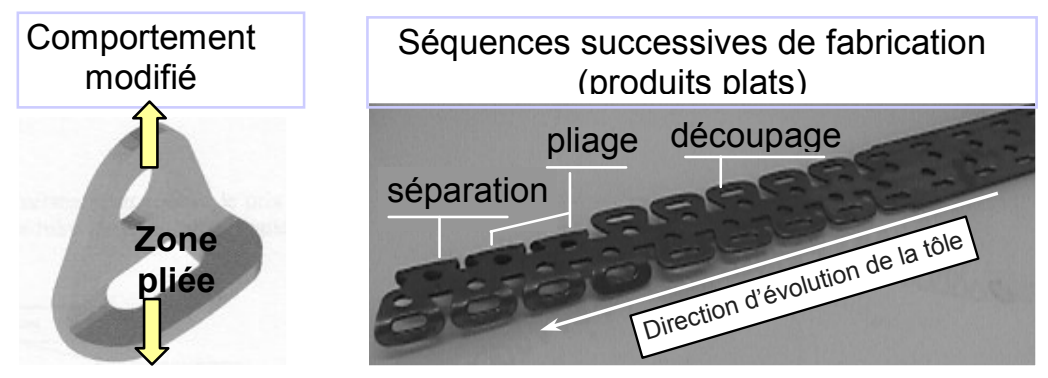

Figure 1. Mise en forme des pièces de sécurité sur presse

La simulation complète de la fabrication des attaches montrée figure 1, est décomposée en opérations élémentaires qui se succèdent. Dans l'étude nous ne retiendrons que le pliage à $90^{\circ}$ par tombé de bord qui sera simulé indépendamment à l'aide du code explicite Abaqus Standard (Hibbit et al., 2005).

\subsection{Le pliage par " tombé de bord"}

\subsubsection{La pièce}

La phase de pliage par «tombé de bord» consiste à plier la tôle par un poinçon qui la plaque à $90^{\circ}$ sur une matrice. Le pliage des pièces de sécurité a été simulé à l'aide d'un modèle tridimensionnel dans lequel on maintient la pièce sur la matrice par un serre-flan tout en la déformant par un poinçon qui se translate verticalement.

Après pliage le matériau est le siège d'un champ de contraintes complexe, essentiellement de traction sur l'extérieur du pli et de compression à l'intérieur. Comme attendu, ces contraintes se concentrent plus près du trou central donnant lieu 
à un endommagement plus fort comme l'a constaté (Bahloul, 2005) lors de ses essais sur presse.

\subsubsection{L'éprouvette}

En vue de validations expérimentales, des éprouvettes plus adaptées aux manipulations (figure 2b) ont été conçues en respectant la caractéristique importante de la pièce réelle qui est le trou oblong central. La largeur utile de l'éprouvette dans la zone centrale, hors trou, est de $12 \mathrm{~mm}$.

Le modèle numérique tridimensionnel de simulation est donné sur la figure $2 \mathrm{a}$ et le maillage de l'éprouvette est représenté sur la figure $2 \mathrm{c}$. Il est constitué d'éléments C4D8, briques à 8 nœuds et 8 points d'intégration. Dans la zone centrale le maillage est raffiné, et il comporte 6 éléments dans l'épaisseur.
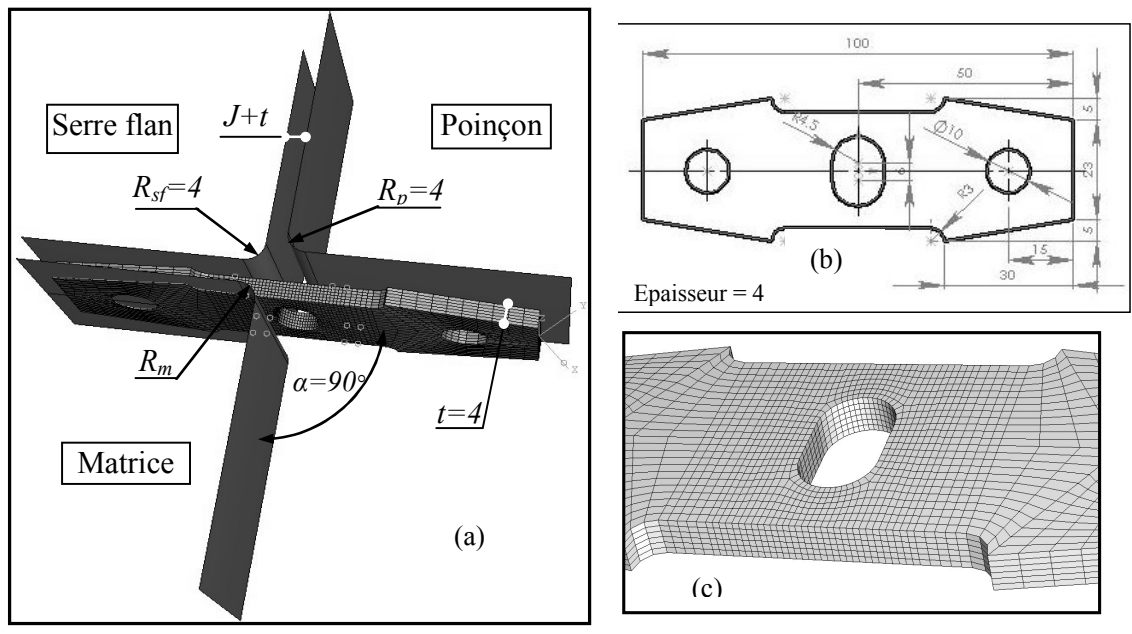

Figure 2. (a) Simulation 3D du pliage (b) éprouvette (c) modèle E.F

Les distributions de la variable d'endommagement isotrope $D$ et des contraintes équivalentes de von Mises sont illustrées sur les figures $3 \mathrm{a}$ et $3 \mathrm{~b}$. Si on rapproche les études faites sur la pièce, on constate que se sont les mêmes zones qui sont les plus sollicitées ce qui conforte la démarche d'étude des éprouvettes en lieu et place des attaches réelles.

Ces exemples montrent clairement la concentration des contraintes et du dommage apporté au matériau dans les parties externes du pli au voisinage du trou oblong. 

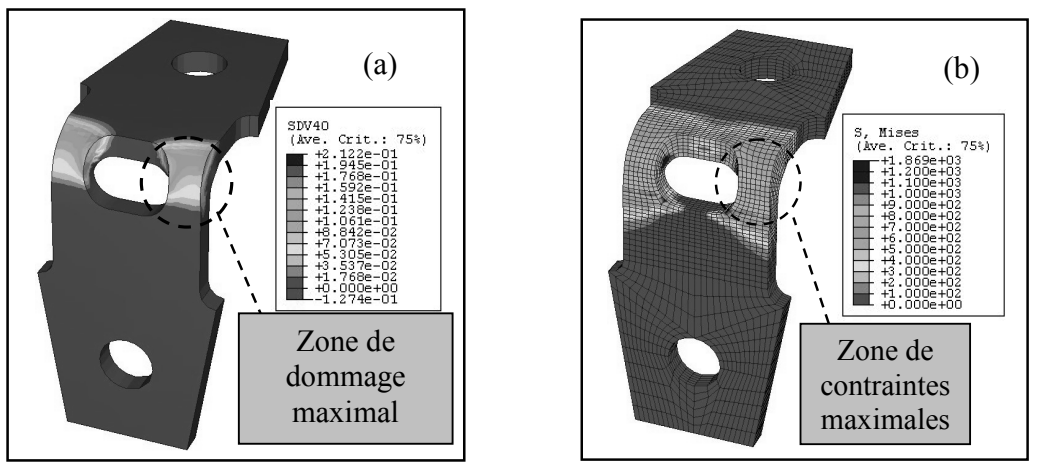

Figure 3. Distributions des contraintes de von Mises (a) et d'endommagement (b) dans les éprouvettes

\section{Identification du comportement du matériau}

Le matériau utilisé pour la fabrication des ferrures d'ancrage est l'acier H.L.E. S500MC livré en bandes de $120 \mathrm{~mm}$ de largeur et de $4 \mathrm{~mm}$ d'épaisseur sous forme de bobines. Les caractéristiques mécaniques module de Young $E$, limite élastique $R_{e}$ et loi d'écrouissage, ont été déterminées sur des éprouvettes plates montées sur une machine de traction. Des essais de charge-décharge effectués sur des éprouvettes à flancs arrondis (Lemaître et al., 1988), ont permis de caractériser la loi d'endommagement par variation du module de Young.

\subsection{Lois de comportement}

L'identification expérimentale de la loi de comportement dans le domaine élastique et plastique permet d'écrire les relations suivantes entre contraintes vraies $(\sigma)$ et déformations vraies $(\varepsilon)$ unidimensionnelles :

$$
\begin{array}{lr}
\sigma=E \varepsilon & \text { si } \sigma \leq R_{e} \\
\sigma=R_{e}+K\left(\bar{\varepsilon}^{P l}\right)^{n} & \text { si } \sigma>R_{e}
\end{array}
$$

$\bar{\varepsilon}^{P l}$ est la déformation plastique cumulée, $K$ est le module d'écrouissage et $n$ l'exposant de cette loi. Leurs valeurs sont respectivement: $K=800 \mathrm{MPa}$ et $n=0,725$.

Les études sont complétées par la caractérisation de l'anisotropie de la tôle à l'aide d'éprouvettes tirées dans la bande à $0^{\circ}, 45^{\circ}$ et $90^{\circ}$ (Mkaddem, 2003).

Dans la suite de cette étude c'est la démarche d'optimisation du procédé et l'aide à la décision pour le choix d'un matériau approprié à une mise en forme par pliage 
qui importe. C'est pourquoi les modèles numériques sont établis à partir d'hypothèses d'isotropie du comportement et de l'endommagement.

\subsection{Dommage}

Les essais de charge-décharge d'éprouvettes à flancs arrondis garantissant la localisation des phénomènes dans la partie centrale conduisent à définir une loi d'évolution de la variable scalaire d'endommagement. Dans l'hypothèse d'un endommagement isotrope, la variable scalaire $D$ caractérisant le dommage est reliée à la déformation plastique équivalente cumulée suivant la relation :

$$
D=f\left(\bar{\varepsilon}^{P l}\right)=1-\frac{E\left(\bar{\varepsilon}^{P l}\right)}{E_{0}}
$$

$E\left(\bar{\varepsilon}^{P l}\right)$ est la valeur du module pour une déformation plastique courante et $E_{0}$ le module du matériau vierge. Conformément aux travaux de Lemaître, on écrit que l'incrément de dommage $\dot{D}$ est relié à l'incrément de déformation plastique équivalente $\dot{\bar{\varepsilon}}^{P l}$ comme suit :

$$
\dot{D}=\frac{D_{C}}{\varepsilon_{R}-\varepsilon_{D}}\left\{\frac{2}{3}(1+v)+3(1-2 v)\left(\frac{\sigma_{H}}{\sigma_{e q}}\right)^{2}\right\} \dot{\bar{\varepsilon}}^{P l}
$$

$D_{C}$ et $\varepsilon_{R}$ sont la valeur du dommage et de la déformation plastique à rupture, $\varepsilon_{D}$ est la valeur de la déformation plastique à partir de laquelle le dommage s'initie. Les valeurs caractéristiques identifiées pour l'acier S500MC sont $\varepsilon_{R}=0,71 \varepsilon_{D}=0.046$ $D_{C}=0,21$.

$\sigma_{e q}$ et $\sigma_{H}$ sont respectivement la contrainte équivalente de von Mises et la contrainte hydrostatique, $v$ est le coefficient de Poisson.

Les relevés expérimentaux ont conduit à identifier une évolution linéaire de la variable d'endommagement $D$ en fonction de $\bar{\varepsilon}^{p l}$. La loi correspondante s'écrit :

$$
D=H_{G} \bar{\varepsilon}^{P l}
$$

avec $H_{G}=D_{C} /\left(\varepsilon_{R}-\varepsilon_{D}\right)$. Pour l'acier utilisé, $H_{G}$ a pour valeur 0,6 . 


\section{Mise en œuvre numérique}

\subsection{Elastoplasticité couplée à l'endommagement}

Le modèle numérique est basé sur un couplage entre une loi élastoplastique et l'endommagement. Le couplage apparaît alors dans la relation contraintesdéformations :

$$
\boldsymbol{\sigma}=(1-D) \mathbf{C}_{0} \boldsymbol{\varepsilon}
$$

$\boldsymbol{\sigma}$ est le tenseur des contraintes de Cauchy et $\boldsymbol{\varepsilon}$ est le tenseur de déformation vraie. $\boldsymbol{C}_{0}$ est l'opérateur de Hooke du matériau vierge. Dans le cadre de la plasticité associée, la fonction de charge $f$ est couplée au dommage isotrope pour s'écrire :

$$
f\left(\sigma_{e q}, \bar{e}^{P l}, D\right)=\frac{\sigma_{e q}}{1-D}-R_{e}-\widetilde{R}\left(\bar{e}^{P l}\right)
$$

$\widetilde{R}=K\left(\bar{e}^{P l}\right)^{n}$ représente la loi d'écrouissage de la relation [1b].

Des procédures rappelées dans (Mkaddem, 2003), permettent de trouver la relation liant les incréments du tenseur des contraintes $\partial \boldsymbol{\sigma}$ et de déformation $\partial \boldsymbol{\varepsilon}$ comme suit :

$$
\partial \boldsymbol{\sigma}=\boldsymbol{K}_{t g D} \partial \boldsymbol{\varepsilon}
$$

La matrice tangente $\boldsymbol{K}_{t g D}$ apparaissant dans cette relation permet le développement d'un algorithme incrémental résolu sous forme implicite par la méthode des éléments finis.

Dans la suite, l'implémentation de l'algorithme dans le code Abaqus Standard a permis l'étude paramétrique des procédés de mise en forme sur presse et plus spécialement des opérations de pliage par «tombé de bord» en vue de leur optimisation.

\subsection{Contacts et loi de frottement}

Les contacts entre les surfaces métalliques en regard se situent entre la tôle et le serre flan et entre la tôle et les outils (poinçon et matrice). Dans le premier cas, ces contacts sont proches d'une adhérence entre surfaces et on a choisi de représenter leur loi d'évolution par la loi de Coulomb avec un coefficient de frottement égal à 0,15 . Dans le cas des contacts glissants entre la tôle et l'outillage, lors des essais on a réalisé une lubrification à l'huile déposée par un pinceau, de sorte que dans la simulation on a adopté une loi de Coulomb avec un coefficient de frottement égal à 0,09 qui est la valeur généralement acceptée dans le cas industriel. La gestion des contacts est effectuée par les sous-programmes internes au code. 


\section{Optimisation du procédé de pliage}

Les procédés de pliage et de découpage mis en œuvre dans la fabrication ont été optimisés de façon indépendante, vu que les objectifs poursuivis ne sont pas exactement les mêmes. Dans le cas du découpage, on cherche à obtenir une partie lisse du bord découpé aussi grande que possible avec une bavure minimale. Dans le cas du pliage, on cherche à minimiser les contraintes résiduelles, l'endommagement et le retour élastique des pièces. Dans la suite, on ne se préoccupera que du procédé de pliage à $90^{\circ}$.

\subsection{Simulation numérique du procédé de pliage}

L'étude a été entreprise sur les éprouvettes trouées sous les deux points de vue expérimental et numérique. La mise en œuvre numérique de l'approche mécanique détaillée en section 3, avec le couplage élastoplasticité/endommagement, est faite dans le code Abaqus Standard où un programme utilisateur UMAT a été développé pour rendre compte du comportement du matériau et de la tôle au cours de sa mise en forme.

La figure 4 est un exemple de résultat obtenu avec les hypothèses simplificatrices évoquées précédemment et une simulation 3D. Une éprouvette en tôle H.L.E. S500MC a été pliée avec un jeu nul $\left(J_{0}\right)$ entre celle-ci et l'outillage en fin d'opération. Les rayons de matrice $\left(R_{m}\right)$ et de poinçon sont de $4 \mathrm{~mm}$, égaux à l'épaisseur. La figure concerne l'évolution de la force de pliage en fonction du déplacement du poinçon. On constate que l'effort prédit est un peu plus grand que dans le cas expérimental lors de la phase de pliage et qu'il chute un peu plus rapidement dans la phase de descente du poinçon. Ces remarques laissent à penser que le matériau est un peu plus «raide » qu'en réalité et qu'il y a peut être un effet d'endommagement par compression qui n'a pas été pris en compte dans le modèle.

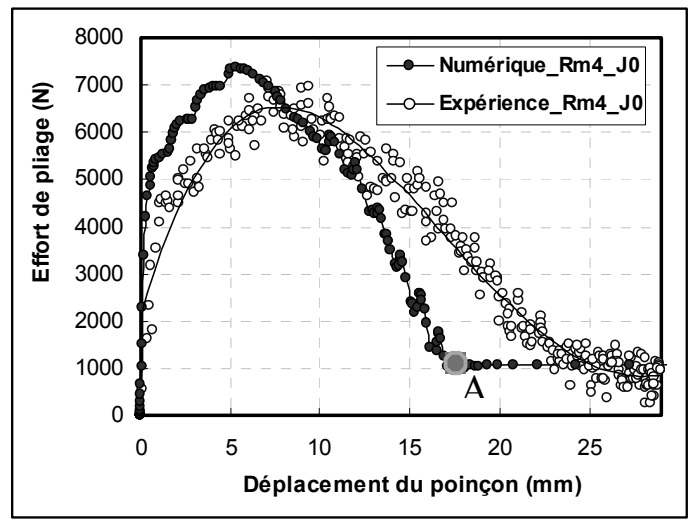

Figure 4. Evolution de la force de pliage en fonction du déplacement du poinçon (Bahloul, 2005) 
De même les effets du frottement du poinçon sur la tôle pliée en fin d'opération paraissent plus «étalés» dans le cas expérimental que dans la modélisation numérique (après le point A sur la figure 4). Néanmoins, les écarts entre mesures et calculs n'excèdent pas 7 à $8 \%$, écarts qui paraissent acceptables pour juger des effets des paramètres du procédé sur le comportement des pièces et caractériser leurs influences sur l'état mécanique et la précision géométrique conditionnant l'usage des composants de sécurité automobile.

Le but de l'optimisation du procédé est alors de déterminer les valeurs optimales des paramètres de l'opération de pliage qui minimiseront les contraintes et l'endommagement du matériau et qui diminueront le retour élastique des pièces une fois formées.

\subsection{Le problème d'optimisation du procédé}

Les pièces de sécurité formées sur presse voient leurs caractéristiques mécaniques et géométriques tributaires des outillages et de la mise en œuvre du procédé. Les paramètres les plus influents ont été identifiés à la suite d'un grand nombre d'essais (Bahloul, 2005). Ils sont de deux ordres :

- les paramètres géométriques définissant les surfaces des outils : rayons de matrice $\left(R_{m}\right)$ et de poinçon $\left(R_{p}\right)$;

- les paramètres de mise en œuvre : le jeu existant entre les outils et la tôle $(J)$, les conditions de lubrification (coefficient de frottement $f$ ).

Dans cette étude, l'épaisseur de la tôle $(t)$ étant fixée par d'autres considérations, elle ne constituera pas une variable d'étude.

Les fonctions objectives retenues pour l'optimisation du procédé sont les deux caractéristiques mécaniques suivantes :

- la contrainte maximale de von Mises $\sigma_{v M \max }$

- la valeur maximale de la variable d'endommagement $D_{\max }$

Le problème d'optimisation se formule donc comme suit :

$$
\begin{aligned}
& \text { Minimiser } \sigma_{v M \max }=\sigma_{v M}\left(R_{m}, R_{p}, J, f\right) \\
& \text { Minimiser } D_{\max }=D_{M}\left(R_{m}, R_{p}, J, f\right)
\end{aligned}
$$

Avec les contraintes :

$$
\begin{aligned}
& R_{m}^{{ }^{\min }}<R_{m}<R_{m}{ }^{\text {max }} \\
& D_{M}{ }^{\text {min }}<D_{M}<D_{M}^{\text {max }}
\end{aligned}
$$

Le coefficient de frottement $f$ peut être considéré comme une variable aléatoire avec une moyenne égale à $f_{0}$ et une variance $\hat{\sigma}^{2}$. Afin de limiter l'étude à ce qui a 
été identifié expérimentalement comme les paramètres principaux de l'opération de pliage, on a supposé que la valeur de $f$ est une constante connue qui ne constitue pas une variable du problème influençant grandement les réponses.

Une analyse dimensionnelle des fonctions et des variables permet de mener l'étude à partir des variables et des fonctions sans dimension suivantes :

$$
\widetilde{R}_{m}=\frac{R_{m}}{t} ; \widetilde{J}=\frac{J}{t} ; \widetilde{\sigma}_{v M}=\frac{\sigma_{v M \max }}{R_{e}} ; \widetilde{D}=D_{\max }
$$

\subsection{Analyse et résolution du problème d'optimisation}

\subsubsection{Méthode des surfaces de réponse}

L'analyse du problème a été réalisée par une méthode dite de surfaces de réponse dont la méthodologie a été formalisée par Myers et Montgomery (2002) Il s'agit ici de développer une méthode robuste d'approximation :

- robuste, parce que les paramètres choisis sont les paramètres importants du procédé qui est assez peu sensible aux variations d'autres variables mal maîtrisées technologiquement ;

- méthode d'approximation, parce qu'il est impossible de trouver des solutions exactes à des processus physiques et techniques aussi complexes que ceux mis en œuvre dans la mise en forme sur presses industrielles. Notons que lorsque la base des résultats provient de simulations, les erreurs de la méthode se cumulent avec celles des calculs numériques.

\subsubsection{Approximation des fonctions objectifs}

En pratique, on procède à une série d'expériences soit physiques soit numériques et on analyse les résultats en construisant des fonctions traduisant les « réponses » recherchées, par des approximations polynomiales. Les réponses correspondent à des jeux de paramètres choisis pour balayer l'espace de conception des expériences. On peut alors réaliser des plans complets ou fractionnaires basés sur des plans d'expériences et analysés par exemple par la méthode de (Taguchi, 1987).

Dans l'analyse des résultats, les approximations sont des régressions linéaires ou quadratiques dans la plupart des cas. On écrit ainsi la réponse $\psi$ sous la forme empirique suivante :

- dans le cas d'une réponse du premier ordre avec deux paramètres $\xi_{1}$ et $\xi_{2}$ :

$$
\hat{\psi}_{1}=a_{0}+a_{1} \xi_{1}+a_{2} \xi_{2}+\varepsilon
$$

- dans le cas d'une réponse complète du second ordre :

$$
\hat{\psi}_{2}=a_{0}+a_{1} \xi_{1}+a_{2} \xi_{2}+a_{3} \xi_{1}^{2}+a_{4} \xi_{1} \xi_{2}+a_{5} \xi_{2}^{2}+\varepsilon
$$


$\varepsilon$ est un terme tenant compte d'une erreur aléatoire par rapport à la valeur exacte de la réponse. Les $\xi_{i}$ sont les variables ou paramètres de conception, les coefficients $a_{i}$ sont les coefficients de régression qui doivent être ajustés pour minimiser l'erreur $\varepsilon$. Ceci est en général fait par une méthode de minimisation d'une erreur quadratique dite de moindres carrés que nous ne détaillerons pas ici.

\subsubsection{Représentation des fonctions approchées}

Après avoir déterminé les fonctions approchées $\hat{\psi}_{l}$ il est possible de les représenter par des surfaces dans le cas de deux variables $\left(\xi_{1} \xi_{2}\right)$ ou, lorsque le nombre de variables est supérieur à 2, de les associer deux par deux pour des représentations tridimensionnelles. Les solutions possédant des formes analytiques, il est possible de rechercher leurs optima par des méthodes d'optimisation classiques.

\subsubsection{Application au pliage}

La présente étude porte sur l'optimisation du procédé de pliage de tôles H.L.E. Les deux paramètres les plus influents qui ont été expérimentalement identifiés sont le rayon de matrice $R_{m}$, qui correspond au rayon du pli, et le jeu $J$ entre la pièce et l'outillage. Lorsque le jeu est négatif, un léger laminage de la tôle se produit dont les effets se traduisent par une diminution du retour élastique.

\subsection{Optimisation de l'opération de pliage}

Les paramètres adimensionnels définis par [12] évoluent de façon discrète dans les plages restreintes suivantes :

$$
0,25 \leq \widetilde{R}_{m} \leq 1,5 \quad-0,15 \leq \widetilde{J} \leq 0,15
$$

Les fonctions réduites $\widetilde{\sigma}_{v M}$ et $\widetilde{D}$ [12] qui sont déterminées numériquement par un plan d'expériences complet, sont approchées par des polynômes complets du second degré.

On cherche à minimiser les contraintes résiduelles et l'endommagement du matériau dans les éprouvettes après pliage. La contrainte équivalente réduite $\widetilde{\sigma}_{v M}$ maximale atteinte pour les 28 valeurs des paramètres réduits $\widetilde{R}_{m}$ et $\widetilde{J}$ dans différents éléments finis, est donnée dans le tableau 1 tiré de (Bahloul et al., 2005) : 
334 REMN - 17/2008. Optimisation des procédés de mise en forme

\begin{tabular}{|c|c|c|c|c|c|c|c|}
\hline $\begin{array}{c}\widetilde{R}_{m} \\
\text { - }\end{array}$ & $-0,15$ & $-0,1$ & $-0,05$ & 0 & 0,05 & 0,1 & 0,15 \\
\hline 0,25 & 2,053 & 1,832 & 1,689 & 1,822 & 1,826 & 1,826 & 1,847 \\
\hline 0,5 & 1,951 & 1,847 & 1,678 & 1,803 & 1,891 & 1,895 & 1,881 \\
\hline 1 & 1,816 & 1,883 & 1,686 & 1,501 & 1,539 & 1,513 & 1,505 \\
\hline 1,5 & 1,996 & 1,818 & 1,724 & 1,433 & 1,453 & 1,430 & 1,447 \\
\hline
\end{tabular}

Tableau 1. Valeurs de la contrainte relative de von Mises

De même, dans le tableau 2 on donne les valeurs maximales du dommage $\widetilde{D}$ atteint dans les éléments finis concernés, pour les 28 valeurs des paramètres du procédé.

\begin{tabular}{|c|c|c|c|c|c|c|c|c|}
\hline $\begin{array}{l}\widetilde{R}_{m} \\
\boldsymbol{\nabla}\end{array}$ & $\widetilde{J}$ & $-0,15$ & $-0,1$ & $-0,05$ & 0 & 0,05 & 0,1 & 0,15 \\
\hline \multicolumn{2}{|c|}{0,25} & 0,99 & 0,793 & 0,589 & 0,496 & 0,793 & 0,794 & 0,892 \\
\hline \multicolumn{2}{|c|}{0,5} & 0,594 & 0,186 & 0,174 & 0,184 & 0,188 & 0,389 & 0,391 \\
\hline \multicolumn{2}{|c|}{1} & 0,135 & 0,141 & 0,142 & 0,147 & 0,147 & 0,151 & 0,151 \\
\hline \multicolumn{2}{|c|}{1,5} & 0,113 & 0,116 & 0,118 & 0,121 & 0,123 & 0,125 & 0,132 \\
\hline
\end{tabular}

Tableau 2. Valeurs du dommage maximal

Dans les deux cas, contraintes et dommage, les matrices d'expériences numériques sont complètes. Dans un souci de représentation optimale, les approximations par surfaces de réponse des deux fonctions découlent de deux approches polynomiales différentes: une approche linéaire et une approche quadratique comme indiqué ci-après :

$$
\begin{aligned}
& \tilde{y}_{1}=a_{0}+a_{1} \widetilde{R}_{m}+a_{2} \widetilde{J} \\
& \tilde{y}_{2}=a_{0}+a_{1} \widetilde{R}_{m}+a_{2} \widetilde{J}+a_{3} \widetilde{R}_{m}{ }^{2}+a_{4} \widetilde{R}_{m} \times \widetilde{J}+a_{5} \widetilde{J}^{2}
\end{aligned}
$$

\subsubsection{Contrainte équivalente de von Mises}

Dans le cas des approximations de $\widetilde{\sigma}_{v M}$, les régressions linéaire ou quadratique conduisent aux expressions : 


$$
\widetilde{\sigma}_{v M 1}=1.9099-0.2145 \widetilde{R}_{m}-0.8761 \widetilde{J}
$$

$\widetilde{\sigma}_{v M 2}=1.8961-0.3942 \widetilde{R}_{m}+0.3500 \widetilde{J}+0.1022 \widetilde{R}_{m}{ }^{2}-1.5090 \widetilde{R}_{m} \times \widetilde{J} \ldots+\ldots+$ $6.8852 \widetilde{J}^{2}$

Les deux surfaces de réponse illustrant les relations [16], [17] sont données sur la figure 5. Par rapport aux valeurs du tableau 1 , les erreurs maximales commises dans l'un ou l'autre cas ont pour valeurs :

- dans le cas linéaire $\varepsilon_{\text {Max linéaire }}=0,277$

- dans le cas quadratique $\varepsilon_{\text {Max quadratique }}=0,1699$

Ce qui correspond à environ $15 \%$ dans le cas linéaire et à $8 \%$ dans le cas quadratique.

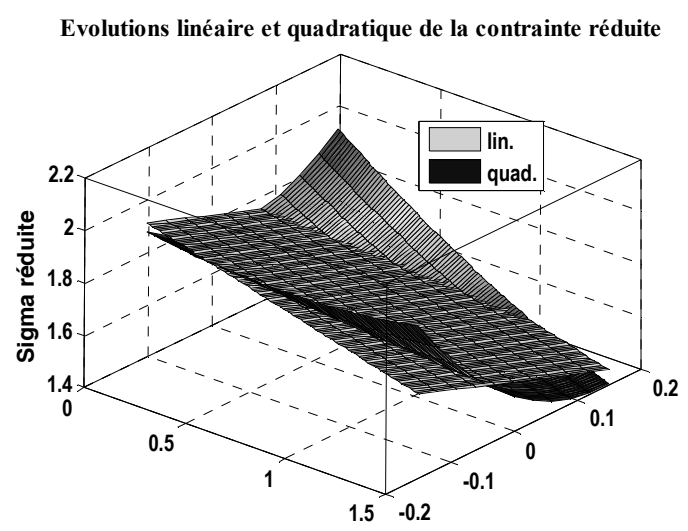

Figure 5. Représentations de $\widetilde{\sigma}_{v M}$ par surfaces de réponse

\subsubsection{Endommagement}

Dans le cas des approximations de la variable d'endommagement $\widetilde{D}$, les régressions linéaire ou multiple conduisent aux deux expressions suivantes :

$$
\widetilde{D}_{1}=0.6968-0.4482 \widetilde{R}_{m}-0.0226 \widetilde{J}
$$

$\widetilde{D}_{2}=1.0391-1.8146 \widetilde{R}_{m}-0.1328 \widetilde{J}+0.7771 \widetilde{R}_{m}{ }^{2}+0.1356 \widetilde{R}_{m} \times \widetilde{J}+$ $7.5914 \widetilde{J}^{2}$ 
Les surfaces correspondantes sur la figure 6 exhibent la même tendance générale décroissante avec des rayons de pliage croissant, ce qui est généralement constaté lors des mises en forme par pliage.

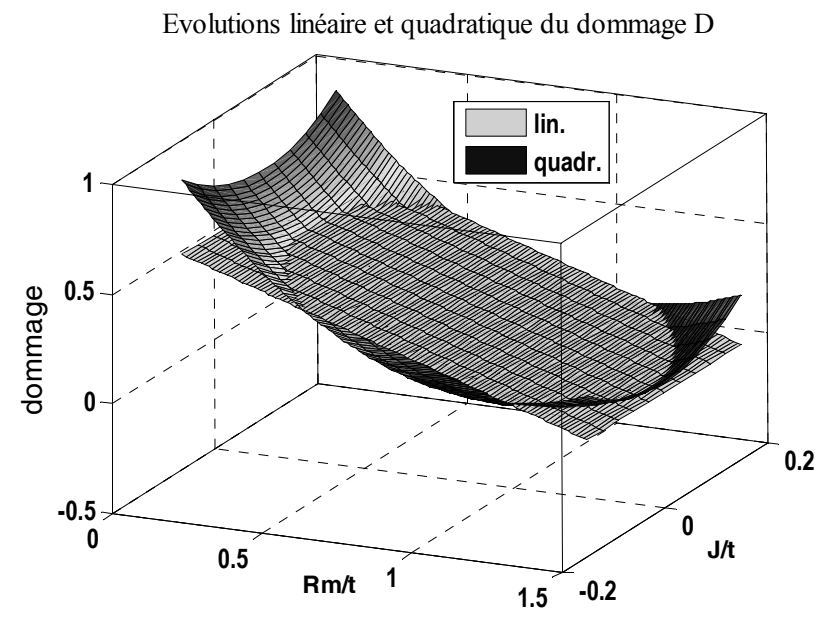

Figure 6. Représentations de $\check{D}$ par surfaces de réponse

Les erreurs que l'on commet dans les approximations du dommage sont élevées dans le cas linéaire et moitié moindre dans le cas quadratique. On a trouvé les valeurs suivantes :

Erreur $_{\text {Max linéaire }}=0,4019$ et Erreur ${ }_{\text {Max quadratique }}=0,2221$

Ce qui correspond environ à $40 \%$ dans le cas linéaire et à $22 \%$ dans le cas quadratique.

D'après la forme de la surface, on constate ici que le rayon de pliage a beaucoup plus d'influence sur la valeur de $\widetilde{D}$ que le jeu et que c'est ce paramètre qui est le plus influent dans le procédé de pliage.

Dans l'un et l'autre cas, contrainte équivalente et endommagement, on constatera que les valeurs minimales sont obtenues pour les plus grands rayons de pliage et les jeux les plus grands. Par contre si on s'intéresse au retour élastique, les conclusions seront inverses, il est d'autant plus grand que $R_{m}$ et $J$ sont grands.

\subsubsection{Retour élastique}

Ce paramètre caractérise la précision géométrique des pièces obtenues après mise en forme. Le retour élastique $\theta$ est mesuré par la différence entre l'angle final de la pièce, identifié par la simulation, et l'angle théorique de $90^{\circ}$. De nombreuses études sur ce sujet ont vu le jour depuis quelques dizaines d'années (Livatyali et al., 2001-2002) mais il est toujours d'actualité de rechercher les paramètres et les formes 
d'outils qui permettront de minimiser ce phénomène lors de mises en forme par déformations plastiques. Par exemple, (I-Nan Choul et al., 1999) se sont attachés à définir des outils qui permettent de réduire le retour élastique en couplant optimisation et analyse par E.F. grâce à une procédure algorithmique basée sur un script en langage Python. C'est une procédure analogue que nous avons adoptée et développée plus loin pour étudier l'influence de différents matériaux sur la mise en forme par pliage.

Pour l'étude du retour élastique par surfaces de réponse, les approximations découlent des résultats obtenus par les simulations 3D. Les fonctions approchées, linéaire et quadratique, ont été trouvées sous les formes polynomiales suivantes :

$$
\begin{aligned}
& \theta_{1}=2,7552+0,776 \widetilde{R}_{m}+7,2436 \widetilde{J} \\
& \theta_{2}=2,3383+1,3464 \widetilde{R}_{m}+3,5065 \widetilde{J}-0,3244 \widetilde{R}_{m}{ }^{2}+4,5995 \widetilde{R}_{m} \times \widetilde{J} \ldots+ \\
& \ldots+24,2310 \widetilde{J}^{2}
\end{aligned}
$$

On a représenté sur la figure 7 son évolution lorsqu'on adopte une approximation linéaire ou quadratique. Les valeurs des erreurs maximales commises dans ces deux cas sont de 0.7933 degrés pour l'approximation linéaire et de 0.3253 degrés pour l'approximation quadratique. Ceci correspond à environ $40 \%$ d'erreur dans le cas linéaire et à $16 \%$ d'erreur dans le cas quadratique.

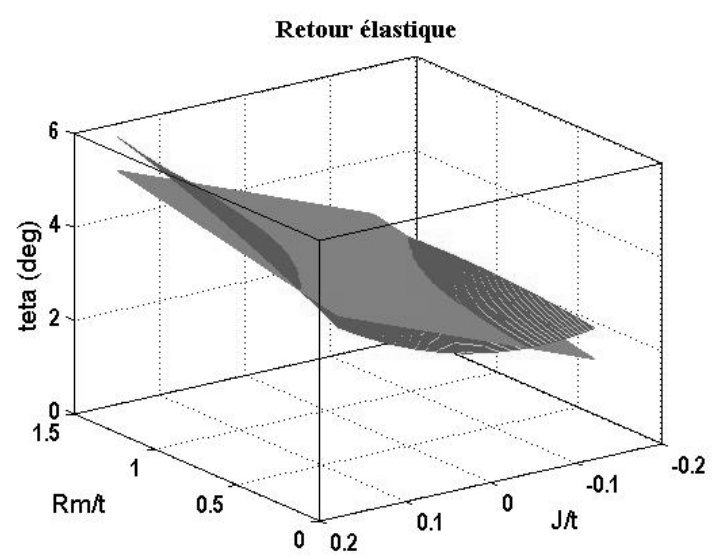

Figure 7. Représentations de l'angle de retour élastique par surfaces de réponse

On constate bien sur la figure que le retour élastique est minimal pour le rayon de pliage minimal et le plus petit jeu, ce qui paraît évident. Le mérite de cette représentation est de montrer l'évolution de la fonction avec $\widetilde{R}_{m}$ et $\widetilde{J}$. On voit que pour des jeux négatifs ( $\widetilde{J}=-0,2)$, le rayon de pliage n'influe pas beaucoup sur la 
valeur de $\theta$ qui est minimale alors que pour des jeux positifs $(\widetilde{J}=0,2)$, le rayon a plus d'influence sur la valeur de $\theta$. Il apparait aussi que c'est le jeu relatif qui est le paramètre qui influe le plus sur l'obtention d'un retour élastique recherché.

Les analyses précédentes montrent qu'il est impossible d'obtenir des pièces par pliage qui présentent à la fois une bonne précision géométrique et un état minimal d'endommagement et de contraintes. Si on veut optimiser les attaches de ceinture en vue de respecter une bonne qualité géométrique et un état de contraintes et d'endommagement acceptable, il est nécessaire d'optimiser le procédé à l'aide d'une fonction multi-objectif.

\subsection{Fonction multi-objectif}

Pour apprécier l'influence du choix des paramètres du procédé sur l'obtention d'une pièce optimale, on a défini une fonction multi-objectif en associant le retour élastique de la tôle et l'endommagement subi par le matériau en fin de pliage. On a normalisé à 1 chacune des deux fonctions approchées $\left(f_{\text {rélas }}\right.$ et $\left.f_{\text {dom }}\right)$ et on a construit une nouvelle fonction $f_{\text {multiobj }}$ en écrivant :

$$
f_{\text {multiobj }}=1 / 2\left(f_{\text {rélas }}+f_{\text {dom }}\right)
$$

Cette fonction pondère les deux fonctions objectifs, retour élastique et dommage, de la même façon en leur attribuant le même coefficient.

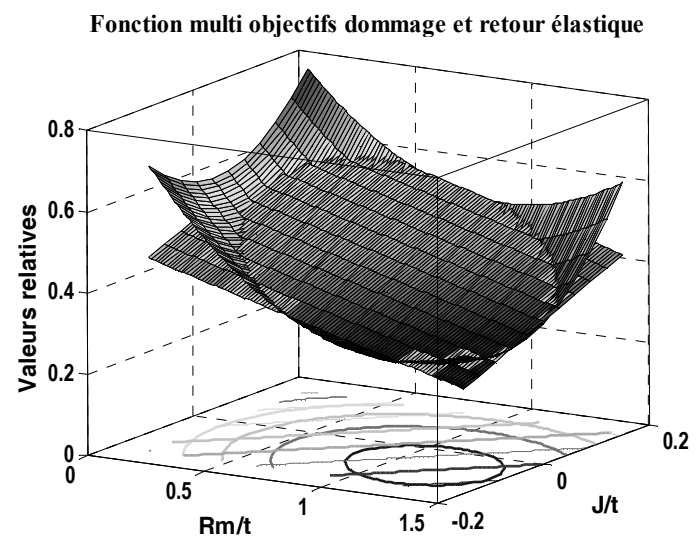

Figure 8. Représentations de la fonction multi-objectif par surfaces de réponse

La représentation des évolutions de cette fonction est donnée par la figure 8. Les expressions des approximations linéaire et quadratique de $f_{\text {multiobj }}$ sont déduites des relations [18-21] et l'approximation linéaire peut y être interprétée comme une valeur moyenne des évolutions réelles. 
Les lignes de contour projetées sur le plan du domaine de faisabilité montrent la zone dans laquelle il faudrait choisir les valeurs des paramètres du procédé afin d'obtenir un endommagement acceptable pour un retour élastique minimisé. Ceci est illustré à la fois sur les figures 8 et 9 . La recherche des valeurs optimales a été faite par un algorithme itératif qui teste les valeurs de la fonction pour en retenir le minimum. La valeur minimale est 0,2629 et elle est obtenue pour un rayon de matrice égal à 1,138 fois l'épaisseur (ici $4,55 \mathrm{~mm}$ ) et un jeu légèrement négatif égal à $-0,066$ fois l'épaisseur (ici $-0,26 \mathrm{~mm}$ ). Un outillage conçu avec ces valeurs conviendrait pour un pliage optimum des pièces, respectant à la fois un endommagement et un retour élastique acceptables.

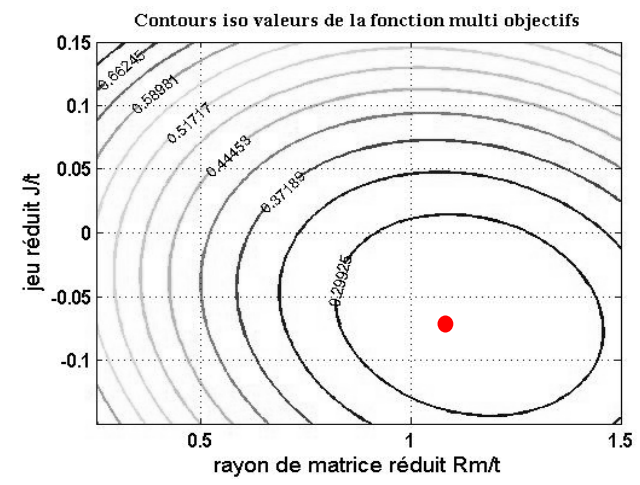

Figure 9. Lignes iso valeurs de la fonction multi-objectif $f_{\text {multiobj }}$

Les erreurs commises sont dans le cas linéaire de 0.2407 et dans le cas quadratique de 0.1068 , ce qui correspond respectivement à une erreur de $30 \%$ et de $13 \%$. Ces erreurs peuvent paraittre excessives mais elles sont dues aux assez brusques variations des données visibles dans les tableaux précédents.

Les études précédentes sont réduites au seul matériau étudié jusqu'ici, l'acier H.L.E. S500MC. Pour un fabricant répondant à un donneur d'ordre, il est aussi important de pouvoir prédire le comportement de pièces produites à partir d'autres matériaux. Il peut aussi vouloir choisir le matériau le plus approprié et pour ces raisons, il est intéressant de pouvoir prédire le comportement en pliage de pièces réalisées dans divers matériaux et c'est ce qu'on entreprend dans la suite.

\section{Prédiction du comportement au pliage en fonction du matériau de la tôle}

\subsection{Matériaux envisagés}

La mise en forme de pièces par pliage concerne une grande variété de tôles dont les épaisseurs sont variables et dont les matériaux ont des caractéristiques mécaniques différentes. Pour un fabricant de pièces par pliage sur presse, il est alors 
intéressant de pouvoir prédire le comportement du matériau afin de prévoir la qualité des pièces en service.

Dans ce but, on a identifié les valeurs de certaines caractéristiques mécaniques des six matériaux reportés dans le tableau 3 :

\begin{tabular}{|c|c|c|c|c|c|c|}
\hline Propriétés & HSLA & $316 \mathrm{~L}$ & $\mathrm{XC} 48$ & $\mathrm{XC60}$ & $\mathrm{A} 1050$ & $\mathrm{~A} 2017$ \\
\hline$t(\mathrm{~mm})$ & 4.0 & 1.2 & 4.0 & 3.5 & 3.0 & 3.0 \\
\hline$E(\mathrm{GPa})$ & 210 & 210 & 210 & 210 & 73 & 73 \\
\hline$v$ & 0.3 & 0.3 & 0.3 & 0.3 & 0.33 & 0.33 \\
\hline$R_{e}(M P a)$ & 560 & 323 & 430 & 407 & 120 & 360 \\
\hline$K(M P a)$ & 1096 & 1280 & 1195 & 1100 & 206 & 722 \\
\hline$n$ & 0.189 & 0.360 & 0.294 & 0.240 & 0.120 & 0.230 \\
\hline$\varepsilon_{D}$ & 0.048 & 0.033 & 0.026 & 0.012 & 0. & 0. \\
\hline$\varepsilon_{R}$ & 0.358 & 0.464 & 0.663 & 0.632 & 0.355 & 0.343 \\
\hline$D_{R}$ & 0.213 & 0.395 & 0.371 & 0.310 & 0.266 & 0.337 \\
\hline
\end{tabular}

Tableau 3. Caractéristiques mécaniques des matériaux retenus

Ces caractéristiques concernent un modèle de loi de comportement $\sigma=k \varepsilon^{n}$ et un modèle d'endommagement avec loi d'évolution du dommage isotrope proposé par Lemaître.

Pour caractériser le matériau dans l'étude paramétrique, on a défini la variable sans dimension $R_{e} / E$ qui prend les valeurs données dans le tableau 4 :

\begin{tabular}{|c|c|c|c|c|c|c|}
\hline matériau & HSLA & $316 \mathrm{~L}$ & $\mathrm{XC} 48$ & $\mathrm{XC60}$ & $\mathrm{A} 1050$ & $\mathrm{~A} 2017$ \\
\hline$\left(R_{e} / E\right) \times 10^{3}$ & 2,667 & 1.538 & 2,048 & 1,938 & 1,644 & 4,932 \\
\hline
\end{tabular}

Tableau 4. Paramètre adimensionnel associé aux matériaux

A partir des caractéristiques mécaniques précédentes, l'étude du procédé de pliage a été entreprise à l'aide de simulations bidimensionnelles. 


\subsection{Modélisation 2D du pliage}

Les calculs tridimensionnels prenant chacun environ 12 heures, on a préféré choisir une modélisation 2D du pliage dans l'hypothèse de déformations planes (figure 10). La discrétisation fait appel à un maillage par des quadrangles dont la taille diminue dans la zone de pliage où les gradients de contraintes et de dommage sont les plus grands. Partant de 4 éléments dans l'épaisseur sous serre-flan, on aboutit à 16 éléments dans la zone du pli. Une procédure automatique a été implémentée dans le code Abaqus Standard, prenant en compte la loi élastoplastique couplée à l'endommagement grâce au sous-programme utilisateur «UMAT » évoqué en section 4. Cette procédure est écrite en langage Python et fait partie d'un «script » qui lance automatiquement en boucle les calculs pour chaque matériau.

Les simulations de pliage des pièces sont conduites dans des conditions d'opérations comparables pour les différentes tôles puisqu'on conserve les mêmes valeurs adimensionnelles $R_{m} / t$ et $\mathrm{J} / \mathrm{t}$ des paramètres de l'opération.
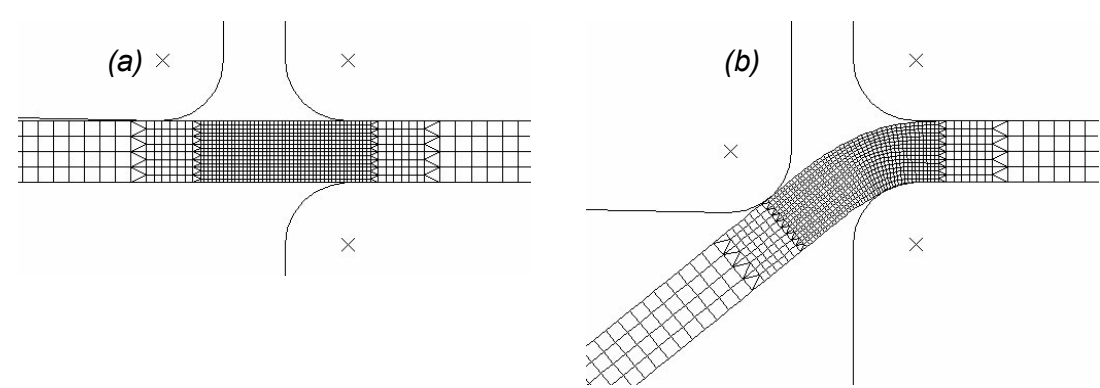

Figure 10. Modèle bidimensionnel du pliage : (a) mise en place (b) pliage en cours

Dans la suite on détaille les résultats de l'étude pour un rayon relatif de pliage $\widetilde{R}_{m}$ invariable égal à 1 . Dans cette hypothèse, on étudie l'influence du jeu relatif $\widetilde{J}$ sur l'endommagement du matériau par un plan d'expériences complet basé sur les résultats numériques des simulations.

Les représentations par surfaces de réponse qui lui correspondent, sont données sur la figure 11a où la fonction dommage $\widetilde{D}$ est représentée sous la forme de facettes reliant les arêtes où sont connues les valeurs provenant des simulations. On y a aussi reporté les valeurs des erreurs commises par l'approximation quadratique représentée par la surface de réponse de la figure $11 \mathrm{~b}$. Sur cette même figure on a reporté l'approximation linéaire de la fonction endommagement. Comme on peut le constater, les erreurs sont assez importantes avec une approximation quadratique, mais ceci ne condamne pas l'intérêt de cette représentation qui donne une tendance pour le choix d'un matériau. 


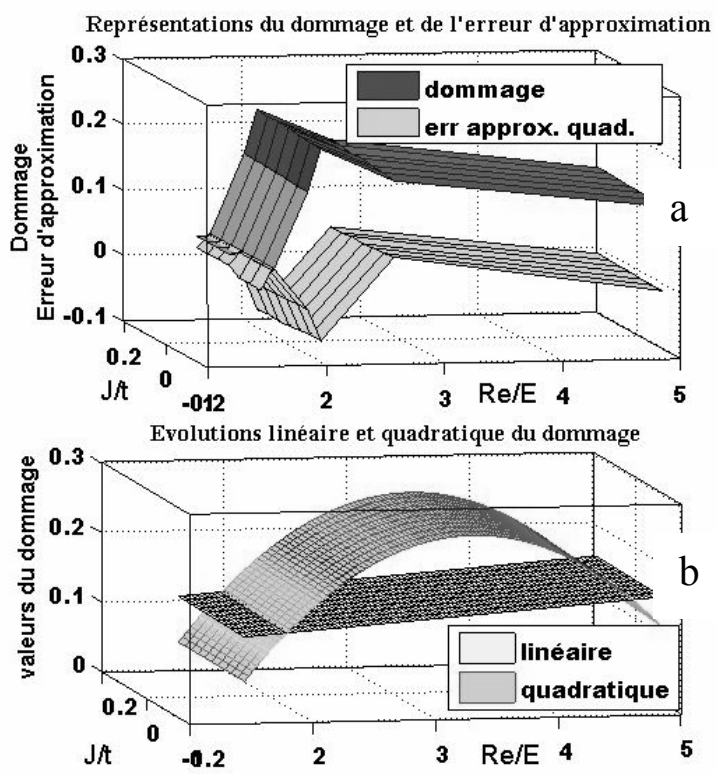

Figure 11. Evolutions du dommage. Surfaces de réponses linéaire et quadratique

En augmentant le degré d'approximation, on a constaté qu'une représentation du troisième degré n'apportait pas d'amélioration au modèle et même qu'elle dégradait la prédiction. Les évolutions qu'on observe sur la figure montrent qu'il n'y a pas d'interactions fortes entre les paramètres et par conséquent que des approximations d'ordres plus élevés ne doivent pas améliorer les prédictions.

L'utilisation de méthodes locales comme le krigeage ou les moindres carrés mobiles pourraient être envisagées pour mieux «suivre » la surface. Dans le cas du krigeage avec un faible nombre de données et un choix de points d'évaluation dépendant de variables discrètes non positionnées aléatoirement (rayons, épaisseurs, etc.), on a remarqué que les erreurs peuvent être élevées.

Pour obtenir avec fiabilité les valeurs optimales des fonctions et des paramètres associés, on a eu recours à une méthode d'optimisation globale fondée sur une stratégie d'évolution dont les mérites ont été comparés dans (Bahloul et al., 2005) (i) à une méthode d'optimisation globale, (ii) à l'utilisation de surfaces de réponse et (iii) à la méthode d'approximation diffuse. 


\section{Optimisation par stratégies d'évolution}

La recherche des valeurs optimales des fonctions objectifs et des paramètres les plus influents dans les procédés de fabrication conduit souvent à des optima locaux. Pour s'en affranchir, on a recours à des méthodes globales souvent basées sur des stratégies d'évolutions.

On peut classer les algorithmes évolutionnaires en programmation évolutionnaire, stratégies d'évolution et algorithmes génétiques. De nombreux travaux ont amené ces techniques à un haut degré d'analyse et de fiabilité (Bäck, 1996 ; Hansen et al., 2001).

La programmation évolutionnaire est développée pour les programmes d'évolution, les stratégies d'évolution $(S E)$ et algorithmes génétiques $(A G)$ sont plus volontiers réservés à la résolution de problèmes d'optimisation.

La différence essentielle entre $(S E)$ et $(A G)$ est l'adaptation de la distribution des mutations. Dans les deux cas, les opérateurs utilisés sont la sélection, la mutation et le croisement. Nous avons emprunté à (Müller, 2002) les analyses qui suivent.

\subsection{Généralités sur l'optimisation par stratégie d'évolution}

\subsubsection{Cas d'un parent et d'un enfant}

Chacun des 2 individus qui constituent une population, est modélisé par un vecteur $\boldsymbol{x}$ avec $n$ composantes réelles $\mathrm{x}_{\mathrm{k}}$ représentant son génotype. Dans ce schéma, on utilise les opérateurs de sélection et de mutation.

- Dans l'étape de mutation, la stratégie génère un descendant représenté par le vecteur $x_{N}^{(g)}$ en ajoutant au parent $x_{A}^{(g)}$ un vecteur aléatoire $\boldsymbol{z}$ avec des composantes normalement distribuées $\boldsymbol{z} \sim \mathcal{N}(\mathbf{0}, \mathbf{I})$ :

$$
x_{N}^{(g)}=x_{A}^{(g)}+\sigma^{(g)} z
$$

- Dans l'étape de sélection, la capacité de survie est déterminée en calculant les valeurs de la fonction objective à la fois pour le parent et le descendant. Dans un contexte de minimisation, celui qui possède la plus petite valeur est choisi pour être le parent de la génération qui suit. La taille globale du pas $\sigma^{(g)}\left(\sigma^{(g)} \in \Re_{>0}\right)$ est un paramètre de stratégie qui est adapté pendant l'optimisation à l'aide d'une règle qui sera définie plus loin.

Le processus peut être décrit comme suit :

$1^{\circ}$-conception d'un descendant

$$
x_{N}^{(g)}=x_{A}^{(g)}+\pi^{(\mathrm{g})} \boldsymbol{z}
$$


$2^{\circ}$ - sélection

$x_{A}^{(g+1)}=\left\{\begin{array}{c}x_{N}^{(g)} \text { si } f\left(x_{N}^{(g)}\right) \leq f\left(x_{A}^{(g)}\right) \\ x_{A}^{(g)} \text { autrement }\end{array}\right.$

$g$ est le compteur des générations, $A$ l'indice du vecteur parent (Ancien), $N$ l'indice du vecteur descendant (Nouveau).

$3^{\circ}$ - si la boucle n'est pas terminée faire $g=g+1$ et retourner en 1 -

\subsubsection{Cas de plusieurs individus}

En plus de ce qui précède, on effectue un croisement entre individus. L'opérateur de recombinaison est en général employé avant la mutation. L'opérateur de recombinaison discret permet $d^{\prime}$ 'échanger des composantes $\mathrm{x}_{\mathrm{k}}{ }^{\text {par1 }}$ et $\mathrm{x}_{\mathrm{j}}{ }^{\mathrm{par} 2}$ des vecteurs qui constituent les deux parents.

Dans le cas de 2 parents $\boldsymbol{x}^{1}$ et $\boldsymbol{x}^{2}$, on constitue un descendant $\boldsymbol{x}^{\prime}$ par recombinaison arithmétique des composantes en écrivant :

$$
x_{i}{ }^{\prime}=\alpha x_{i}^{1}+(1-\alpha) x^{2}{ }_{i} \quad \alpha \in[0,1] \text { et } i=1, \ldots n .
$$

Dans les $S E$, les choix des paramètres stratégiques comme l'étendue de la population, la taille du pas de la mutation ou le schéma de sélection, sont cruciaux pour le comportement et les propriétés de convergence de la stratégie.

Une attention particulière doit être portée à l'adaptation de la taille du pas pendant le déroulement de l'optimisation. Divers mécanismes pour l'adaptation de la taille globale du pas $T G P$ ont été proposés comme :

- l'adaptation cumulative qui consiste à adapter la $T G P$ en accumulant des informations sur les sélections non locales au cours d'une séquence de générations ;

- l'adaptation par matrice de covariance qui adapte la distribution de la mutation.

Dans ce dernier cas, on mute les vecteurs paramétriques à l'aide d'un vecteur normalement distribué $\boldsymbol{z} \sim \mathcal{N}(\mathbf{0}, \mathbf{I})$ qu'on transforme par une application linéaire représentée par une matrice carrée $n \times n \sqrt{C}$. Le vecteur $\sqrt{C} \boldsymbol{z}$ est alors $\mathcal{N}(\mathbf{0}, \boldsymbol{C})$ distribué. La matrice $\boldsymbol{C}$ est la matrice de covariance de la distribution de mutation. Le vecteur paramétrique $\boldsymbol{x}_{\mathrm{k}}{ }^{(\mathrm{g}+1)} \mathrm{du} \mathrm{k}^{\text {ième }}$ individu dans la génération $(g+1)$ est calculé par la relation :

$$
\boldsymbol{x}_{\mathrm{k}}^{(\mathrm{g}+1)}=\langle x\rangle_{\mu}^{(g)}+\sigma^{(\mathrm{g})} \sqrt{C} \boldsymbol{z}_{\mathrm{k}} \quad \mathrm{k}=1, \ldots, \lambda
$$


$\langle x\rangle_{\mu}^{(g)}$ est la recombinaison intermédiaire des $\mu$ vecteurs paramétriques sélectionnés dans la génération $(g)$, c'est-à-dire le centre de masse des $\mu$ meilleurs individus. On le calcule par :

$$
\langle x\rangle_{\mu}^{(g)}=\frac{1}{\mu} \sum_{i \in I_{\text {sel }}^{(g)}} x_{i}^{(g)}
$$

Ici $I_{\text {sel }}^{(g)}$ est l'ensemble des indices ( $\mu$ au total) des individus sélectionnés dans la génération $(\mathrm{g})$.

Le mécanisme d'adaptation comprend deux aspects :

- l'adaptation de la matrice de covariance $\boldsymbol{C}^{(\mathrm{g}+1)}$

- l'adaptation de la taille globale du pas de mutation.

Ces deux mécanismes ne sont pas détaillés ici mais peuvent être trouvés dans (Müller, 2002). Dans les applications envisagées, ces stratégies seront mises en œuvre pour optimiser le procédé de pliage.

\subsection{Application à l'optimisation du procédé de pliage}

On applique la méthode précédente, avec adaptations de la matrice de covariance et de la taille globale du pas, au pliage par tombé de bord. On cherche les valeurs optimales des paramètres réduits $\widetilde{R}_{m}$ et $\widetilde{J}$ qui minimisent la fonction multi-objectif de la section 5.5 [24]. Les bornes de variation sont toujours les mêmes : $0,25 \leq \widetilde{R}_{m}$ $\leq 1,5$ et $-0,15 \leq \widetilde{J} \leq 0,15$. L'algorithme de la stratégie d'évolution (Hansen, 2001) est développé sous le code MATLAB (Matlab, 2005).

Le minimum de la fonction qui a pour valeur 0,2629 est obtenu pour les valeurs des paramètres $\widetilde{R}_{m}{ }^{\text {opt }}=1,1391$ et $\widetilde{J}^{\text {opt }}=-0,0646$. Ces valeurs sont à rapprocher de celles données en section 5.5 pour constater qu'elles sont quasi-identiques.

La figure 12 montre l'évolution de la valeur de la fonction $f_{\text {multiobj }}$ avec le nombre d'itérations (figure 12a) et les évolutions des valeurs des paramètres vers leurs optima respectifs (figure 12b). Comme on l'a indiqué précédemment, la taille globale du pas $\sigma^{(\mathrm{g})}$ (Sigma sur la figure) diminue régulièrement au fur et à mesure que la fonction objectif se stabilise près de son optimum.

Les courbes sur les figures $12 \mathrm{a}-\mathrm{b}$ montrent que l'optimum est rapidement atteint, après une centaine d'évaluations et ce, en une à deux secondes de calculs sur un ordinateur de performances courantes. 
Cet exemple montre que la stratégie d'évolution adoptée est un moyen intéressant pour des prédictions fines des valeurs optimales des paramètres qui interviennent dans des processus de fabrication complexes.

On objectera ici que les approximations polynômiales représentatives des phénomènes étudiés ne présentent pas de grandes variations des gradients et que n'apparaissent que des optima facilement identifiables par des méthodes classiques. On a pu cependant constater dans d'autres cas de surfaces plus «tourmentées » que ces méthodes fournissaient rapidement des solutions globales satisfaisantes.
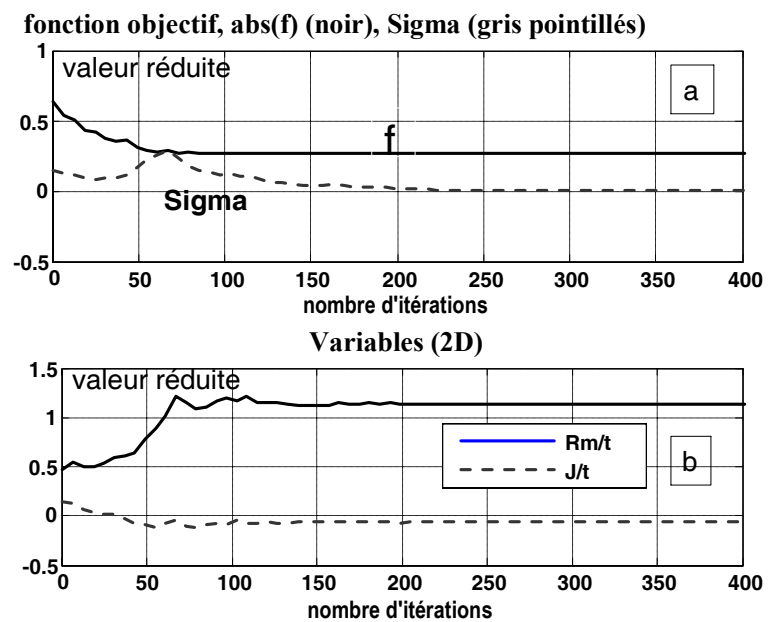

Figure 12. Évolutions de la fonction et des paramètres en fonction du nombre d'itérations

\section{Conclusion}

Les procédés de mise en forme de pièces minces sur presses pour des applications de sécurité automobile mettant en jeu des procédés de découpage et de pliage, induisent des champs de contraintes et d'endommagement qui doivent être minimisés. La prise en compte de l'endommagement de la matière doit impérativement être englobée dans la loi de comportement élastoplastique des aciers à haute limite élastique utilisés dans les fabrications de ferrures d'ancrage. La loi d'endommagement continu de Lemaître couplée à la plasticité est une approche fiable pour la modélisation du comportement des pièces lors de leur mise en forme. Elle peut être introduite dans les codes industriels par l'intermédiaire d'algorithmes «utilisateurs » comme les sous-programmes UMAT du code E.F. Abaqus. Les prédictions des réponses mécaniques, contraintes et endommagement du matériau ainsi que la prédiction des valeurs du retour élastique n'étant pas accessibles aux calculs analytiques, on procède à des expériences numériques à partir de plans d'expériences. Par des techniques de régression, il est alors possible de définir des approximations polynomiales qu'on représente sous forme de surfaces de réponse. 
L'analyse des résultats des simulations numériques peut alors être faite directement à partir des graphes, ou par des techniques d'optimisation classiques, pour fournir les valeurs optimales des paramètres des procédés.

Dans le cas du pliage par «tombé de bord», deux résultats contradictoires apparaissent lors de la recherche des optima, qui montrent que pour minimiser le retour élastique il faudrait un petit rayon de pliage et un jeu minimal dans l'outillage alors que pour minimiser contraintes et dommage il faudrait un rayon de pliage et un jeu dans l'outillage maximaux. Une fonction multi-objectif est alors définie qui pondère également les deux exigences précédentes et la surface de réponse qui lui est associée permet de déterminer les valeurs optimales du jeu et du rayon de matrice.

Enfin, le choix de matériaux les plus appropriés pour de nouvelles fabrications a été basé sur l'identification des caractéristiques mécaniques de sept matériaux métalliques différents. Des variables adimensionnelles caractérisant le matériau et le procédé ayant été définies, l'optimisation du procédé de pliage a été conduite par une stratégie d'évolution qui fournit le minimum global de la fonction objectif et les valeurs des paramètres du procédé.

\section{Remerciements}

Les auteurs expriment leur reconnaissance à la Société Devillé SA Z.I. de Beauregard 49150 à Baugé (France) pour son support technique et financier.

\section{Bibliographie}

Bäck T., Evolutionary Algorithms in Theory and practice, Oxford University Press, 1996.

Bahloul R., Ben Eléchi S., Dal Santo P., Potiron A., "Optimisation of the L-bending process A comparison between Response Surface, Moving least squares and Evolution Strategies Methods", $1^{\text {st }}$ Invited Cost 526 Conf. Automatic Process Optimisation in Materials Technology, Morschach, Switzerland, Proceedings, May 30-31, 2005, p. 120-131.

Bahloul R., Optimisation du procédé de pliage sur presse de pièces en tôle à haute limite élastique, Thèse de Doctorat, 5 décembre 2005, Ecole Nationale Supérieure d'Arts et Métiers, CER d'Angers.

Bahloul R., Dal Santo P., Potiron A., "Optimisation of process parameters in wiping-die bending operation in order to minimise stresses and Lemaitre damage", International Conference on Metal Forming, ESAFORM 2005, Cluj-Napoca Romania, p. 159-162.

Hambli R., Potiron A., Modélisation et simulation du découpage des tôles, Techniques de l'Ingénieur, 2004 BM 7 505, p. 1-18.

Hambli R., Potiron A., "Finite element modelling of sheet-metal blanking operations with experimental verification", Journal of Materials Processing Technology, 2000, p. 257-265.

Hansen N., 2001/3. cmaes.m, Version 2.31. http://www.bionik.tu-berlin.de/user/niko

Hansen N., Ostermeier A., "Completely derandomised self-adaptation in evolution strategies", Evolutionary Computation, vol. 9, n² 2, 2001, p. 159-195. 
348 REMN - 17/2008. Optimisation des procédés de mise en forme

Hibbit Karlsson, Sorensen Inc, 1080 Main Street Pawtucket RI 02860-4847 USA ABAQUS Standard User's manual, version 6.52005 .

I-Nan Chou, Chinghua Hung, "Finite element analysis and optimisation of springback reduction", International Journal of Machine Tools and Manufacture, 39, 1999, p. 517-536.

Lemaître J., Chaboche J., Mécanique des Matériaux Solides, Dunod, Paris, 1988, p. 343-444.

Lemiale V., Picart P., Meunier S., "Numerical modelling of blanking process for thin sheet metal parts", Proceedings of the $5^{\text {th }}$ Intern. Conf. on Material Forming ESAFORM Cracovie 14-17 avril 2002, p. 559-562.

Livatyali H., Wu H.C., Altan T., "Prediction and elimination of spring back in straight flanging using computer aided design methods", Part 1: Experimental investigations, Journal of Material Processing Technology, vol. 117, 2001, p. 262-268.

Livatyali H., Wu H.C., Altan T., "Prediction and elimination of spring back in straight flanging using computer aided design methods", Part 2: F.M. predictions and tools design, Journal of Material Processing Technology, vol. 120, 2002, p. 348-354.

Matlab 7 version 7.0.4 (R14), The MathWorks, 3 Apple Hill Drive, Natick, MA 01760- 2098 USA.

Mkaddem A., Expérimentation et simulation du pliage H.L.E. Prévision du comportement en service des pièces pliées, Thèse de Doctorat, 16 décembre 2003, Ecole Nationale Supérieure d'Arts et Métiers, CER d'Angers.

Müller S.D., Bio-inspired optimisation algorithms for engineering applications, $\mathrm{PhD}$. Swiss Institute of Technology Zürich 2002.

Myers R.H., Montgomery D.C., Response Surface Methodology; Process and Product Optimization Using Designed Experiments, $2^{\text {nd }}$ edition Wiley series in probability and statistics 2002, J. Wiley \& Sons New York.

Taguchi G., System of Experimental Design: Engineering Method To Optimize Quality And Minimize Costs, UNIPUB/Kraus International White Plains N.Y. 1987.

Wang C.T., Kinzel G.L., Altan T., "Mathematical modelling of plane-strain bending of sheet and plate", Journal of Material Processing Technology, vol. 39, 1993, p. 279-304. 